\title{
OCCURRENCE OF MITRAL VALVE INSUFFICIENCY IN CLINICALLY HEALTHY BEAGLE DOGS
}

\author{
Károly VÖRÖS ${ }^{1 *}$, Viktória SzILVÁSI ${ }^{1}$, Ferenc MANCZUR ${ }^{1}$, Ákos MÁTHÉ$^{1}$, \\ Jenő ReICZIGEL ${ }^{2}$, Ingo NOLTE ${ }^{3}$ and Stephan HuNGERBÜHLER ${ }^{3}$ \\ ${ }^{1}$ Department and Clinic of Internal Medicine and ${ }^{2}$ Department of Biomathematics and \\ Informatics, Faculty of Veterinary Science, Szent István University, P.O. Box 2, \\ H-1400 Budapest, Hungary; ${ }^{3}$ Small Animal Clinic, University of Veterinary Medicine \\ Hanover, Hanover, Germany
}

(Received 28 June 2015; accepted 28 October 2015)

\begin{abstract}
Chronic degenerative valve disease (CDVD) is the most common cardiac disease in dogs, usually resulting in mitral valve insufficiency (MVI). The goal of this study was to investigate the occurrence of MVI in clinically healthy Beagle populations. A total of 79 adult healthy Beagles (41 females and 38 males; age: $5.6 \pm 2.7$ years, range 1.4 to 11.7 years) were examined. The diagnosis of MVI was based on the detection of a systolic murmur heard above the mitral valve, and was confirmed by colour flow Doppler (CFD) echocardiography. Systolic mitral valve murmurs were detected in 20/79 dogs (25.3\%), of them 11 males and 9 females with no statistically significant gender difference $(P=0.6059)$. The strength of the murmur on the semi-quantitative $0 / 6$ scale yielded intensity grade $1 / 6$ in 10 dogs, grade $2 / 6$ in 4 dogs, and grade $3 / 6$ in 6 dogs. Mild to moderate MVI was detected by CFD in all these 20 dogs with systolic murmurs. Of them, $17 \mathrm{dogs}$ had mild and 3 demonstrated moderate MVI, showing 10-30\% and 30-50\% regurgitant jets compared to the size of the left atrium, respectively. The age of dogs with MVI was $7.1 \pm 2.3$ years, which was significantly different from that of dogs without MVI $(5.1 \pm 2.7$ years, $\mathrm{P}=0.0029)$. No significant differences in body weight $(\mathrm{P}=0.1724)$ were found between dogs with MVI $(13.8 \pm 2.8 \mathrm{~kg})$ and those without MVI $(12.8 \pm 3.0 \mathrm{~kg})$. Mitral valve disease causing MVI is relatively common in Beagle dogs, just like in other small breed dogs reported in the literature.
\end{abstract}

Key words: Heart, mitral valve, insufficiency, murmur, echocardiography, dog, Beagle

Although the Beagle is the standard and a widely used dog in clinical experiments and teaching, there is scant information on the prevalence of cardiac diseases in this breed (Patterson, 1968; Marsboom et al., 1971). Only a few papers can be found in the literature on the occurrence of congenital heart diseases (Detweiler and Patterson, 1965; Patterson, 1968). Of the congenital valvular dis-

*Corresponding author; E-mail: Voros.Karoly@aotk.szie.hu; Phone: 0036 (1) 478-4132; Fax: 0036 (1) 478-4137 
orders, pulmonic stenosis and pulmonic valve dysplasia have been mentioned (Patterson, 1968; Patterson et al., 1981; Fujii et al., 2007). A case of atrial septal defect associated with angiostrongylosis has been described by Estèves et al. (2004). Ventricular septal defects and conotruncal malformations have been reported by Diez-Prieto et al. (2009) in a Beagle family.

Chronic degenerative valve disease (CDVD) is the most common cardiac disease of dogs, resulting in mitral valve insufficiency (MVI) in about $60 \%$ of CDVD cases. Both the mitral and tricuspid valves are involved in $30 \%$ and the tricuspid valve alone in 10\% of the cases (Kienle and Thomas, 2002; Olsen et al., 2010; Borgarelli and Buchanan, 2012). CDVD (also having several other names in the literature) mainly occurs in older dogs of small breeds, and males show clinical signs at a younger age compared to females (Thrusfield et al., 1985; Olsen et al., 2010; Borgarelli and Buchanan, 2012). The Cavalier King Charles Spaniel is the most predisposed breed, and dogs of this breed demonstrate MVI at a younger age than those of other breeds (Häggström et al., 1992; Swenson et al., 1996). Dachshunds, Poodles, Spaniels, and Chihuahuas are also regarded as predisposed breeds (Olsen et al., 1999; Häggström et al., 2009; Borgarelli and Buchanan, 2012). Recently, mitral valve disease has been reported to be a relatively common disorder in Norfolk Terriers as well (Trafny et al., 2012). The inherited nature of the disease has been demonstrated in Cavalier King Charles Spaniels and in Dachshunds (Swenson et al., 1996; Olsen et al., 1999; French et al., 2012). Despite the wide use of Beagles for research and teaching, surprisingly little information is available in the literature on the occurrence of mitral valve insufficiency (MVI) in Beagles (Buchanan, 1977; Thrusfield et al., 1985; Chetboul et al., 2007). Nakayama et al. (1994) reported the presence of mitral regurgitation in normal Beagle dogs without mentioning the auscultatory findings.

In our previous study which aimed to compare the diagnostic value of traditional and electronic stethoscopes in recognising cardiac murmurs in 33 healthy, adult Beagle dogs, mitral murmurs were heard and MVI was diagnosed by colour flow Doppler (CFD) echocardiography in 7 (21\%) of these dogs (Szilvási et al., 2013). This proportion seemed to be a relatively high occurrence rate. However, the number of dogs examined in that study had been relatively low. Therefore, our goal was to expand that previous study by including more dogs to reveal clinically healthy individuals with subclinical MVI in a larger population and to demonstrate the possible relationship of MVI with gender and aging as it has been shown in several other breeds and cited above.

\section{Materials and methods}

Animals

A total of 79 adult individuals older than 1.4 years were chosen from clinically healthy Beagle populations without gender selection. Inclusion criteria 
were the absence of symptoms associated with cardiac or respiratory insufficiency. Therefore, two dogs of the originally recruited 81 animals were excluded because of upper respiratory signs revealed by the physical examination. Fortythree out of the 79 dogs were recruited randomly from the population of Aurigon-Toxicoop Research Center Ltd. (Dunakeszi, Hungary), an institute performing pharmaceutical studies. Fourteen dogs were used from another farm breeding Beagles for research purposes (WO-Be Ltd., Budapest, Hungary). Eight dogs were included from the Department of Animal Nutrition, Faculty of Veterinary Science, Szent István University, Hungary, and 14 dogs from the university canine population of the University of Veterinary Medicine Hanover, Germany. Thirty-three out of the 79 dogs of the present study had formed the study material of our previous work with another goal, i.e. comparing the diagnostic value of traditional and sensor-based electronic stethoscopes (Szilvási et al., 2013). The study was approved by the Ethics Committee of the Faculty of Veterinary Science as well as by the relevant authorities.

\section{Physical examinations}

Physical examination was done on all dogs with special regard to the cardiorespiratory system including thoracic auscultation and percussion as described earlier (Vörös et al., 2012).

Cardiac auscultation was performed by four examiners (KV, SH, VSZ, ÁM) in the different groups, where KV took part in all examinations. Dogs were auscultated in a quiet room for about $5 \mathrm{~min}$. Auscultation was done by the participating clinicians independently of each other first with their regularly used traditional stethoscopes (Rappaport-Sprague, Supraphone, Wilhelm Hasselmeyer Medical Development GmbH, Germany as well as Littman TM Classic II, 3M, Health Care, St. Paul, MN, USA). Then, an electronic sensor-based stethoscope (Welch Allyn Meditron Master Elite Stethoscope System, The Stethoscope Welch Allyn Corp., Skaneateles Falls, NY, USA) was used by each investigator. Cardiac murmurs were described and their intensity was graded on the $0 / 6$ scale according to previous descriptions (Sisson and Ettinger, 1999; Kvart and Häggström, 2002; Lundin and Kvart, 2010; Vörös et al., 2011). After cardiac auscultation, a consultation on the absence/presence and grading of a murmur was made and the final classification on murmur characteristics was concluded based on the findings obtained with either stethoscope and on the agreement of at least two investigators where KV was always included.

\section{Echocardiography}

Echocardiography was performed with a Mindray M5-Vet ultrasound system equipped with a 2-4 MHz phased-array transducer (Shenzhen, P. R. China) and with an ESAOTE Megas GPX ultrasound system, using a 5.0-7.5 MHz 
phased-array transducer (ESAOTE S.p.A, Firenze, Italy), as well as with a Vivid 7 GE ultrasound instrument (General Electrics Healthcare, Milwaukee, USA) by applying 3.5-7.0 MHz electronic sector transducers. M-mode, two-dimensional (2D) and Doppler echocardiographic examinations were done by one of the authors (FM, KV, or $\mathrm{SH}$ ) as used in routine, clinical echocardiography (Boon et al., 1983; Brown and Gaillot, 2008; Vörös et al., 2009; Bonagura and Fuentes, 2015).

During the echocardiographic procedures, the M-mode technique was applied especially for the end-diastolic and end-systolic measurements of left ventricular dimensions as well as for the calculation of fractional shortening (FS\%), whilst the two-dimensional method was used with particular attention to measure aortic, left atrial, and ventricular diameters, to calculate left atrial : aortic ratio as well as to detect potential thickening and prolapse of the mitral and tricuspid valve leaflets (Olsen et al., 2003; Chetboul and Tissier, 2012).

Spectral and colour flow Doppler (CFD) echocardiographic modalities were mainly applied to confirm the presence or absence of mitral and tricuspid valve regurgitation as the major indicator of chronic degenerative valvular disease (CDVD). For detecting valvular insufficiencies of all cardiac valves, CFD and spectral Doppler echocardiography were done in the right parasternal long axis and short axis as well as in the left parasternal long axis views. Spectral Doppler, i.e. pulsed wave Doppler (PWD) and continuous wave Doppler (CWD) echocardiographic measurements were done in these views to determine the maximal flow velocities across the cardiac valves or intracardiac shunts. For CFD, the Doppler gain settings were adjusted to the maximum level but without background artefacts (Tou et al., 2006). A semi-quantitative method with CFD was applied to estimate the amount of regurgitant blood flow caused by MVI as described earlier (Pedersen et al., 1999a; Chetboul and Tissier, 2012; Vörös et al., 2012). During the reviewing process of the recordings, the presence or absence of a regurgitant jet was determined. Then, the area of the largest jet was compared to the size of the left atrium. Based on jet size, four groups were used: no jet or very small jet $(0-10 \%)$, small jet $(>10-30 \%)$, medium jet $(>30-50 \%)$, and large jet $(>50 \%)$. Based on this classification, the grade of MVI was assigned as being minimal, mild, moderate, or severe, respectively, as described by Pedersen et al. (1999a). A similar method was used for the semi-quantitative estimation of tricuspid valve, pulmonic valve, and aortic valve insufficiencies comparing the area of the largest jet to the size of the right atrium in right parasternal long axis view and to the left ventricular outflow tract (tricuspid and aortic insufficiency, respectively), and to the size of the right ventricular outflow tract in the right parasternal short axis view (pulmonic insufficiency). 


\section{Further examinations}

Electrocardiography. Electrocardiograms in standard bipolar (Einthoven) leads were performed in right lateral recumbency for 3-min leads with the main goal to diagnose the presence of cardiac arrhythmias.

Laboratory tests. Total blood count as well as total protein and albumin concentrations were measured in dogs that demonstrated cardiac murmurs during auscultation, for detecting possible anaemic and/or blood viscosity alterations which might be responsible for heart murmurs.

\section{Statistical methods}

For the continuous variables (age, body weight) mean $\pm \mathrm{SD}$ and range are reported, and group comparisons were made by Student's $t$-test. For the comparison of categorical variables (occurrence of MVI, grade of murmur) Fisher's exact test was applied, and odds ratio (OR) with $95 \%$ confidence interval (CI) is reported. The odds ratio (OR) and Fisher's exact test were used for the comparison of our Beagle data to other 14 breeds reported by Thrusfield et al. (1985) regarding the occurrence of MVI. Statistical analysis was done by the statistical program R 3.0.2 (R Core Team, 2013).

\section{Results}

Animals

During the study, 79 adult Beagle dogs were examined, 38 of them being intact females, 3 castrated females, 32 intact males, and 6 castrated males, i.e. altogether 41 females $(51.9 \%)$ and 38 males $(48.1 \%)$ constituted the study group. Their age was $5.6 \pm 2.7$ years (range: $1.4-11.7$ years). The age of the males was $6.3 \pm 3.0$ years and that of the females $5.0 \pm 2.3$ years $(\mathrm{P}=0.0319)$. The mean body weight of the dogs was $13.0 \pm 3.0 \mathrm{~kg}$ (males $14.5 \pm 2.9 \mathrm{~kg}$ and females $11.7 \pm 2.3 \mathrm{~kg}, \mathrm{P}=0.0001$ ).

Physical examination did not reveal any abnormalities of the respiratory organs and other organ systems, i.e. all dogs were found to be clinically healthy. During the ECG examinations, 59 (75\%) dogs had physiological respiratory (sinus) arrhythmia, and during this finding, auscultation did not detect a pulse deficit. The ECG results proved to be otherwise negative in all dogs. Blood count values as well as total protein and albumin concentrations were within the normal ranges in all participating dogs.

\section{Presence of mitral insufficiency based on cardiac auscultation} and echocardiography

Of the 79 dogs, a systolic murmur of grade $1 / 6$ to $3 / 6$ was heard in 20 $(25.3 \%)$ above the point of maximal intensity of the mitral valve, i.e. in the 5 th 
intercostal space, at the apex of the heart, just above the sternum (10 with intensity grade $1 / 6,4$ with grade $2 / 6$, and 6 with grade $3 / 6$ ). In one dog (with a grade $1 / 6$ systolic murmur) mid-systolic clicks were also heard.

Mild to moderate MVI was detected by CFD in all 20 dogs (25.3\%) with the systolic murmurs mentioned above. Of them, 17 dogs had mild and three demonstrated moderate MVI, showing $10-30 \%$ and $30-50 \%$ regurgitant jets compared to the size of the left atrium, respectively. CWD echocardiography was also performed in 14 out of the 20 dogs with MVI detected by CFD and PWD echocardiography. In these dogs CWD revealed maximal regurgitant flow velocities from 4.0 to $4.5 \mathrm{~m} / \mathrm{sec}$. No Doppler echocardiographic signs of altered diastolic function were found in any of the dogs with MVI based on pulsed wave examination of the transmitral flow.

During Doppler echocardiography, 10/10 dogs with a grade 1/6 murmur had mild MVI, whilst 3/4 dogs with grade 2/6 demonstrated mild and 1/4 moderate MVI. Among the six dogs with a grade 3/6 murmur, Doppler echocardiography revealed 4/6 dogs with mild and 2/6 with moderate MVI.

No abnormal echocardiographic values were measured by M-mode and two-dimensional echocardiography in any of the 79 dogs with or without MVI. Mild thickening and mitral valve prolapse were seen during two-dimensional echocardiography in 1/20 dogs with MVI detected by Doppler echocardiography. This dog had a grade $2 / 6$ systolic murmur above the mitral valve.

\section{Auscultatory and echocardiographic findings of the other cardiac valves}

In 9/79 (11.4\%) of the dogs, a systolic murmur above the tricuspid valve of grade $1 / 6$ to grade $2 / 6$ was heard during auscultation. No tricuspid valve insufficiency (TVI) was detected by Doppler echocardiography of these individuals. All of these dogs with TVI also had mild MVI during Doppler echocardiography and a grade $2 / 6$ to $3 / 6$ systolic murmur above the mitral valve simultaneously, which was one grade stronger that the murmur above the tricuspid valve. As such, these tricuspid murmurs were considered to be murmurs conducted from the mitral valve.

CFD echocardiography demonstrated only mild tricuspid valve insufficiency (TVI) in $1 / 79(1.3 \%)$ of the dogs. As no murmurs were auscultated in this dog, either above the mitral or the tricuspid valve, TVI was considered to be physiological (trivial).

Mild (physiological) pulmonic valve insufficiency without any murmurs was detected by CFD and PWD echocardiography in 5/79 dogs (6.3\%).

\section{Relationship of mitral valve insufficiency (MVI) with gender, age, and body weight}

MVI occurred in 11/20 (55\%) of the male and in 9/20 (45\%) of the female dogs. This difference between genders was not statistically significant $(\mathrm{P}=$ $0.6059)$. 
The age of dogs with MVI was $7.1 \pm 2.3$ years, which was significantly different from that of dogs without MVI $(5.1 \pm 2.7$ years; $\mathrm{P}=0.0029)$.

Table 1 demonstrates the distribution of dogs with and without MVI according to age and the strength of the mitral valve murmur. The presence of MVI (based on the physical examination and Doppler echocardiographic findings) significantly increased with age $(\mathrm{P}=0.0205)$. As demonstrated in this table, the strength of the murmur tended to increase with age, although this increase was not significant.

Table 1

Distribution of dogs $(n=79)$ with and without MVI according to age and the strength of the mitral valve systolic murmur

\begin{tabular}{lcccc}
\hline & \multicolumn{4}{c}{ Age groups } \\
\cline { 2 - 5 } & $1.0-3.0$ years & $3.1-6.0$ years & $6.1-9.0$ years & $9.1-12.7$ years \\
\hline Number of dogs, total & 17 & 31 & 21 & 10 \\
\hline No. of dogs without murmur & 16 & 25 & 11 & 7 \\
No. of dogs with murmur grade $1 / 6$ & 1 & 3 & 6 & 0 \\
No. of dogs with murmur grade $2 / 6$ & 0 & 2 & 1 & 1 \\
No. of dogs with murmur grade 3/6 & 0 & 1 & 3 & 2 \\
\hline No. of dogs with MVI & $1(5.9 \%)$ & $6(19.4 \%)$ & $10(47.6 \%)$ & $3(42.9 \%)$ \\
\hline
\end{tabular}

The odds ratio (OR) indicated a significantly higher risk of mitral valve insufficiency in our Beagle population compared to 14 other breeds studied by Thrusfield et al. (1985). This was valid both for males (OR $=10.0 ; 95 \%$ confidence interval: 4.4 to $21.2 ; \mathrm{P}<0.0001)$ and females ( $\mathrm{OR}=9.3 ; 95 \% \mathrm{CI}: 3.9$ to 20.3; $\mathrm{P}<0.0001)$.

No significant differences $(\mathrm{P}=0.1724)$ in body weight were found between our dogs with MVI $(13.8 \pm 2.8 \mathrm{~kg})$ and without MVI $(12.8 \pm 3.0 \mathrm{~kg})$.

\section{Discussion}

Buchanan (1977) reported the occurrence of valvular disease in dogs based on pathology or clinical signs. In his study, only 134 Beagles, i.e. $2.6 \%$ of the 5,222 dogs examined, were found to have chronic valvular disease. Thrusfield et al. (1985) found 7/84 (8.3\%) dogs with mitral insufficiency (valvular incompetence) among their male Beagles, based on clinical signs. Our data regarding the male individuals, i.e. 11/38 (28.9\%), reflect a considerably higher occurrence rate of MVI. This is also reflected by the difference of their $(\mathrm{OR}=2.18)$ and our $(\mathrm{OR}=10.0)$ odds ratios. Interestingly, Thrusfield et al. (1985) did not mention any female Beagles in their report. Dennis et al. (1978) studied the tech- 
nical possibilities of M-mode echocardiography in 9 adult Beagles, 4 with and 5 without mitral murmurs, to assess the applicability of M-mode echocardiography for monitoring cardiac valvular dynamics. However, they did not report data on epidemiology and clinical findings of CDVD. Chetboul et al. (2007) selected 12 asymptomatic Beagles with left apical systolic murmur (grade $1 / 6$ or 2/6) and echocardiographic signs of spontaneous mitral valve disease from a population of 450 adult Beagle dogs for a clinical trial without mentioning the occurrence rate, gender and age distribution of the disease.

In our previous study aimed at comparing the diagnostic capacities of traditional and sensor-based electronic stethoscopes, we 'accidentally' detected 7 dogs with MVI (21\%) among 33 clinically healthy Beagles (Szilvási et al., 2013). Based on our current results on 79 dogs, we demonstrated in an acceptably large Beagle population that MVI is a quite common disorder also in Beagles, as 20 $(25.3 \%)$ dogs showed mild to moderate mitral valve regurgitation confirmed by Doppler echocardiography. All these dogs had a systolic murmur above the mitral valve. The MVI occurrence rate found in this study is even higher than in our above-mentioned preliminary results. Our present findings are comparable with most of the observations on clinically healthy populations of other predisposed breeds (Table 2). Olsen et al. (1999) reported on 190 Dachshunds of breeders' families with a mean age of $5.4 \pm 2.9$ years and found mitral murmurs in 55 (29\%). In another study, 131 client-owned, clinically healthy Dachshunds aged more than 2 years were examined clinically as well as with 2D and CFD echocardiography by Olsen et al. (2003). Of them, 17 (13\%) had a mitral murmur of grade 1 to 3 on the 0/6 scale. However, by CFD, they found $71(53.4 \%)$ dogs with a mitral regurgitation jet larger than $10 \%$ of the left atrial area (i.e. at least with mild mitral insufficiency). Pedersen et al. (1999b) detected a systolic, left apical (mitral valve) murmur in 45/153 (29\%) Cavalier King Charles Spaniels between one and 10 years of age and being free of any other clinical signs of cardiovascular diseases but they did not report the number of these dogs with regard to Doppler echocardiographic findings. Trafny et al. (2012) have recently reported the occurrence of mitral valve disease in $23(48 \%)$ out of 48 overtly healthy, more than 6 years old Norfolk Terriers with the presence of a systolic, left apical, i.e. mitral valve murmur. A reasonable high number, i.e. $18(38 \%)$ of the dogs did not have a murmur but was considered to have mitral valve disease based on the echocardiographic findings (mitral valve thickening or prolapse) and CFD evidence of mild mitral valve regurgitation.

During MVI, a systolic click might be present with or without a murmur as it occurred in one of our cases (Ware, 2007). MVI due to chronic degenerative mitral valve disease results in a systolic murmur in most cases, best heard above the mitral valve (Ware, 2007). However, no murmur might occur in mild cases (Pedersen et al., 1999a; Trafny et al., 2012). The murmur intensity usually correlates with the severity of MVI, although rarely the murmur can be mild or even 
inaudible in case of severe regurgitation and heart failure (Häggström et al., 1995; Desjardins et al., 1996). In the present study, in all dogs with MVI confirmed by CFD, systolic mitral murmurs ranging from $1 / 6$ to $3 / 6$ could be heard during auscultation at least by two of the four examiners and using both acoustic and sensor-based electronic stethoscopes. Recently, a four-level murmur grading scheme has been suggested instead of the classical six-grade scale to estimate the strength of cardiac murmurs caused by myxomatous mitral valve disease (Ljungvall et al., 2014). This classification 'compresses' the six-grade scale into four categories: soft (previously $1 / 6$ and 2/6), moderate (3/6), loud (4/6), and thrilling $(5 / 6$ and $6 / 6)$ murmurs. By applying this system, $14(70 \%)$ of our 20 cases with mitral murmurs could be categorised as mild and $6(30 \%)$ cases as moderate intensity murmurs. Usually even mild mitral murmurs reflect cardiologically significant MVI which often progresses in intensity and to a stage with echocardiographic and clinical signs of cardiac insufficiency (Häggström et al., 1992; Olsen et al., 2003; Drut et al., 2015). Nakayama et al. (1994) reported mitral regurgitation in $3(15 \%)$ dogs of 20 normal, young (less than 2 years old) Beagles based on CFD but they did not give data on the size of the jets during their CFD examinations. Their PWD and CWD results yielded a mean peak velocity of $0.9 \mathrm{~m} / \mathrm{sec}$, which was considered to be physiological (or trivial) as it was far below the generally accepted lower limit of about $4.5 \mathrm{~m} / \mathrm{sec}$ peak velocity (Kienle and Thomas, 2002). In human patients, Berger et al. (1989) concluded that a jet with a peak velocity of more than $4.0 \mathrm{~m} / \mathrm{sec}$ may indicate abnormal mitral regurgitation.

Table 2

Occurrence of mitral valve insufficiency (MVI) in clinically healthy dogs of various breeds

\begin{tabular}{|c|c|c|c|c|c|}
\hline Breed & $\begin{array}{l}\text { Number } \\
\text { of dogs } \\
\text { examined }\end{array}$ & $\begin{array}{l}\text { Age } \\
\text { of the population } \\
\text { (years) }\end{array}$ & $\begin{array}{c}\text { Dogs with } \\
\text { MVI } \\
\text { (number } \\
\text { and percentage) }\end{array}$ & $\begin{array}{l}\text { Examination } \\
\text { methods }\end{array}$ & Authors \\
\hline Beagle & 79 & $\begin{aligned} \mathrm{x} & =5.6 \\
\mathrm{SD} & = \pm 2.7\end{aligned}$ & $20(25.3 \%)$ & A, E, SPD, CFD & $\begin{array}{l}\text { Vörös et al., } \\
2015 \\
\text { (this study) }\end{array}$ \\
\hline Dachshund & 190 & $\begin{aligned} \mathrm{x} & =5.4 \\
\mathrm{SD} & = \pm 2.9\end{aligned}$ & $55(29 \%)$ & A & $\begin{array}{l}\text { Olsen et al., } \\
1999\end{array}$ \\
\hline Dachshund & 131 & $>2$ & $\begin{array}{c}17(13 \%) \\
71(53.4 \%)\end{array}$ & $\begin{array}{c}\text { A } \\
\text { E, CFD }\end{array}$ & $\begin{array}{l}\text { Olsen et al., } \\
\quad 2003\end{array}$ \\
\hline $\begin{array}{l}\text { Cavalier King } \\
\text { Charles Spaniel }\end{array}$ & 61 & $\begin{array}{c}x=6.4 \\
S D=2.8\end{array}$ & $32(52 \%)$ & A & $\begin{array}{l}\text { Häggström et } \\
\text { al., } 1992\end{array}$ \\
\hline Norfolk Terrier & 48 & $\begin{array}{c}>6 \\
\mathrm{IQR}=7-10\end{array}$ & $\begin{array}{l}23(48 \%) \\
41(85 \%)\end{array}$ & $\begin{array}{c}\mathrm{A} \\
\mathrm{E}, \mathrm{CFD}\end{array}$ & $\begin{array}{c}\text { Trafny et al., } \\
2012\end{array}$ \\
\hline
\end{tabular}

Abbreviations: $\mathrm{x}=$ mean; SD: standard deviation; IQR: interquartile range; A: auscultation; $\mathrm{E}$ : echocardiography; SPD: spectral Doppler; CFD: colour flow Doppler 
Doppler echocardiography is considered to be the proper tool of diagnosing MVI and estimating its grade semi-quantitatively as has been done in this study and reported earlier (Pedersen et al., 1999a; Muzzi et al., 2003; Szilvási et al., 2013). Spectral Doppler echocardiography provides measurement values for maximal flow velocities of the regurgitant blood flow, but the semi-quantitative CFD technique alone is accepted for screening examinations (Olsen et al., 1999; Olsen et al., 2003; Trafny et al., 2012).

Although cardiac auscultation alone can be used as a screening tool for diagnosing MVI, it might miss mild cases not producing mitral valve murmurs but demonstrated by CFD (Pedersen et al., 1999a; Lundin and Kvart, 2010; Trafny et al., 2012). We considered a regurgitant jet size of at least $>10 \%$ of the left atrial area for the diagnosis of a cardiologically significant MVI, in agreement with other authors (Pedersen et al., 1999a; Olsen et al., 2003; Drut et al., 2015). However, further investigations are considered to be necessary to establish the criteria for differentiation between physiological and mild MVI (Drut et al., 2015; Fonfara, 2015).

Thickening and prolapse of the mitral valve are also suggested to be included in the echocardiographic parameters to diagnose chronic mitral valve disease in dogs (Pedersen et al., 1999b; Olsen et al., 2003). However, early, mild cases might show equivocal or no alterations which might be the reason while we had these signs only in one of our dogs (Terzo et al., 2009).

A systolic murmur above the tricuspid valve can be the result of tricuspid insufficiency caused by either chronic degenerative disease of this valve itself, by tricuspid dysplasia or by pulmonary hypertension (Ware, 2007). Pulmonary hypertension is estimated traditionally by the presence of tricuspid valve insufficiency (TVI) with a maximal regurgitation velocity of $>3.0 \mathrm{~m} / \mathrm{sec}$ and or by pulmonary insufficiency with a maximal regurgitation velocity of $>2.2 \mathrm{~m} / \mathrm{sec}$ as stated by Bonagura and Fuentes (2015). No clinical signs and echocardiographic evidences supported the presence of pulmonary hypertension in our 9 cases with TVI. Rather, a secondary murmur conducted from the affected mitral valve or a non-significant, physiological murmur might have occurred in these dogs. The mild pulmonic insufficiency of our 5 cases can be physiological (Brown and Gaillot, 2008). Nakayama et al. (1994) found in 20 normal Beagles pulmonary $(75 \%)$, mitral $(15 \%)$, and aortic regurgitations $(10 \%)$ with low peak flow velocities, and they considered them as physiological.

No statistically significant differences were seen in this study regarding the occurrence of MVI between female and male individuals. This observation is in accordance with previous reports and might suggest that MVI occurs similarly in the two genders but might progress faster in males, whilst early and mild cases might be present in a similar proportion of males and females (Häggström et al., 1992; Swenson et al., 1996; Borgarelli and Buchanan, 2012). 
It is well known that the occurrence rate of MVI murmurs caused by CDVD increases with age as it was seen in our population as well (Häggström et al., 1992; Olsen et al., 1999; Häggström et al., 2009; Borgarelli and Buchanan, 2012). In the present study, we have included clinically healthy adult Beagles on a random basis from 1.4 to 11.7 years old with a mean age of 5.6 years. The relatively high proportion of older dogs in our population might explain the considerably high occurrence rate of MVI which was also reflected by the age distribution of the affected individuals. The strength of the murmur tended to increase with age, although this was not significant which might be due to the relatively low number of cases within the age groups. However, in Beagle populations kept for research or teaching, animals are usually culled at a younger age, which might explain the scant number of previous publications on the occurrence of MVI in this breed.

No correlation was found between the prevalence of MVI and body weight in the present study. Pedersen et al. (1999b) reported a negative correlation between the degree of MVI to body weight.

\section{Limitations of the study}

No pathological examinations were possible for valvular morphology and for postmortem diagnosis of CDVD in our cases: However, MVI is a consequence of CDVD, and it is generally considered that MVI is mainly due to chronic valvular degeneration at least in miniature and small breeds. It is accepted in the literature to diagnose MVI by auscultation and/or by CFD echocardiography as stated above. In our study, semi-quantitative estimation of the regurgitation jet was mainly used to ultimately diagnose MVI as described earlier (Pedersen et al., 1999a; Muzzi et al., 2003). Although this method has certain technical problems, it has been accepted for clinical applications as other Doppler techniques, like the PISA method, are more cumbersome and also have inherent limitations (Ware, 2007; Chetboul and Tissier, 2012).

MVI can also be caused by congenital mitral valve dysplasia or can be a sequel of dilation of the mitral ring as well as abnormal conformation of the left ventricle in cases of dilated cardiomyopathy in dogs (Ware, 2007; Brown and Gaillot, 2008). In our cases, no clinical or echocardiographic signs of these disorders were present, therefore it can be suggested that MVI was due to CDVD.

Although different ultrasound machines were used in the study due to the different locations of our examinations which might have influenced the echocardiographic (especially the colour Doppler) studies, all echocardiographic instruments were capable of producing reliable images and measurement values.

The number of patients and their gender as well as their age distribution seemed to be enough for estimating the prevalence of this disorder in clinically healthy Beagle dogs. Nevertheless, regular screening can be proposed in other Beagle colonies to recognise MVI at least by auscultation and more precisely even 
by echocardiography to further explore the true presence of this condition. No follow-up of our cases with MVI was possible because of the usual selling or adopting of the dogs after a certain age/time in colonies used for research or teaching.

\section{Final conclusions}

MVI, possibly due to degenerative mitral valve disease, was found to be relatively common in clinically healthy Beagle populations, and older animals were more frequently affected. Investigations of a presumable genetic origin seem to be reasonable also in Beagles, just as studying the occurrence of MVI among Beagles kept as companion animals. The latter might also allow to follow the progression of CDVD in this breed as well.

\section{Acknowledgements}

This research was supported by the 9877-3/2015/FEKUT grant of the Hungarian Ministry of Human Resources. The authors express their thanks to István Novák (A-TRC Ltd.) and Gábor Bognár (Wo-Be Ltd.), as well as to Zsolt Péter and Dániel Fekete (Faculty of Veterinary Science, Szent István University) for their help during the examinations. This study forms part of the PhD study of Viktória Szilvási (Doctoral School of FVS, SZIU). The author Károly Vörös was supported by ERASMUS and DAAD (the German Academic Exchange Program) when performing part of the study in Hanover.

\section{References}

Berger, M., Hecht, S. R., Van Tosh, A. and Lingam, U. (1989): Pulsed and continuous wave Doppler echocardiographic assessment of valvular regurgitation in normal subjects. J. Am. Coll. Cardiol. 13, 1540-1545.

Boon, J., Wingfield, W. E. and Miller, C. W. (1983): Echocardiographic indices in the normal dog. Vet. Radiol. 24, 214-221.

Borgarelli, M. and Buchanan, J. W. (2012): Historical review, epidemiology and natural history of degenerative mitral valve disease. J. Vet. Cardiol. 14, 93-101.

Bonagura, J. D. and Fuentes, W. L. (2015): Echocardiography. In: Nyland, T. G. and Mattoon, J. S. (eds) Small Animal Diagnostic Ultrasound. 3rd edition. Elsevier Saunders, St. Luis. pp. 217-331.

Brown, D. and Gaillot, H. (2008): Heart. In: Penninck, D. and d'Anjou, M. A. (eds) Atlas of Small Animal Ultrasonography. Ames, Blackwell Publishing. pp. 151-216.

Buchanan, J. W. (1977): Chronic valvular disease (endocardiosis) in dogs. Adv. Vet. Sci. Comp. Med. 21, 75-106.

Chetboul, V. and Tissier, R. (2012): Echocardiographic assessment of canine degenerative mitral valve disease. J. Vet. Cardiol. 14, 127-148.

Chetboul, V., Lefebvre, H. P., Sampedrano, C. C., Gouni, V., Saponaro, V., Serres, F., Concordet, D., Nicolle, A. P. and Pouchelon, J-L. (2007): Comparative adverse cardiac effects of pimobendan and benazepril monotherapy in dogs with mild degenerative mitral valve disease. A prospective, controlled, blinded, and randomized study. J. Vet. Intern. Med. 21, 742-753. 
Dennis, M. O., Nealeigh, R. C., Pyle, R. L., Gilbert, S. H., Lee, A. C. and Miller, C. W. (1978): Echocardiographic assessment of normal and abnormal valvular function in Beagle dogs. Am. J. Vet. Res. 39, 1591-1598.

Desjardins, V. A., Enriquez-Sarano, M., Tajik, A. J., Bailey, K. R. and Seward, J. B. (1996): Intensity of murmurs correlates with severity of valvular regurgitation. Am. J. Med. 100, 149-156.

Detweiler, D. K. and Patterson, D. F. (1965): The prevalence and types of cardiovascular disease in dogs. Ann. N. Y. Acad. Sci. 127, 481.

Diez-Prieto, I., García-Rodríguez, B., Ríos-Granja, A., Cano-Rábano, M., Peña-Penabad, M. and Pérez García, C. (2009): Cardiac conotruncal malformations in a family of beagle dogs. J. Small Anim. Pract. 50, 597-603.

Drut, A., Ribas, T., Floch, F., Franchequin, S., Freyburger, L., Rannou, B., Cadoré, J. L. and Bublot, I. (2015): Prevalence of physiological heart murmurs in a population of 95 healthy young adult dogs. J. Small Anim. Pract. 56, 112-118.

Estèves, I., Tissier, D., Dandrieux, J., Polack, B., Carlos, C., Boulanger, V., Muller, C., Pouchelon, J. L. and Chetboul, V. (2004): Reversible pulmonary hypertension presenting simultaneously with an atrial septal defect and angiostrongylosis in a dog. J. Small Anim. Pract. 45, 206-209.

Fonfara, S. (2015): Listen to the sound: what is normal. J. Small Anim. Pract. 56, 75-76.

French, A. T., Ogden, R., Eland, C., Hemani, G., Pon-Wong, R., Corcoran, B. and Summers, K. M. (2012): Genome-wide analysis of mitral valve disease in Cavalier King Charles Spaniels. Short communication. Vet. J. 193, 283-286.

Fujii, Y., Yamane, T., Orito, K., Osamura, K. and Wakao, Y. (2007): Increased chymase-like activity in a dog with congenital pulmonic stenosis. J. Vet. Cardiol. 9, 39-42.

Häggström, J., Hansson, K., Kvart, C. and Swenson, L. (1992): Chronic valvular disease in the Cavalier King Charles Spaniel in Sweden. Vet. Rec. 131, 549-553.

Häggström, J., Höglund, K. and Borgarelli, M. (2009): An update on treatment and prognostic indicators in canine myxomatous mitral valve disease. J. Small Anim. Pract. 50, 25-33.

Häggström, J., Kvart, C. and Hansson, K. (1995): Heart sounds and murmurs: changes related to severity of chronic valvular disease in the Cavalier King Charles Spaniel. J. Vet. Intern. Med. 9, 75-85.

Kienle, R. D. and Thomas, W. P. (2002): Echocardiography. In: Nyland, T. G. and Mattoon, J. S. (eds) Small Animal Diagnostic Ultrasound. 2nd edition. W. B. Saunders, Philadelphia. pp. 354-424.

Kvart, C. and Häggström, J. (2002): Cardiac Auscultation and Phonocardiography in Dogs, Horses and Cats. TK i Uppsala AB, Uppsala. pp. 13-20.

Ljungvall, I., Rishniw, M., Porciello, F., Ferasin, L. and Ohad, D. G. (2014): Murmur intensity in small-breed dogs with myxomatous mitral valve disease reflects disease severity. J. Small Anim. Pract. 55, 545-550.

Lundin, T. and Kvart, C. (2010): Evaluation of the Swedish breeding program for Cavalier King Charles spaniels. Acta Vet. Scand. 52, 54. Published online 2010 Sep 23. doi: 10.1186/1751-0147-52-54

Marsboom, R., Spruyt, J. and Van Ravestyn, C. (1971): Incidence of congenital abnormalities in a beagle colony. Lab Anim. 5, 41-48.

Muzzi, R. A., de Araújo, R. B., Muzzi, L. A., Pena, J. L. and Silva, E. F. (2003): Regurgitant jet area by Doppler color flow mapping: quantitative assessment of mitral regurgitation severity in dogs. J. Vet. Cardiol. 5, 33-38.

Nakayama, T., Wakao, Y., Takiguchi, S., Uehi, M., Tanaka, K. and Takahashi, M. (1994): Prevalence of valvular regurgitation in normal Beagle dogs detected by color Doppler echocardiography. J. Vet. Med. Sci. 56, 973-975.

Olsen, L. H., Fredholm, M. and Pedersen, H. D. (1999): Epidemiology and inheritance of mitral valve prolapse in Dachshunds. J. Vet. Intern. Med. 13, 448-456. 
Olsen, L. H., Häggström, J. and Petersen, H. D. (2010): Myxomatous mitral valve disease. In: Ettinger, S. J. and Feldman, E. C. (eds) Textbook of Veterinary Internal Medicine. Diseases of the Dog and the Cat. 7th edition. Elsevier Saunders, St. Louis. pp. 1299-1315.

Olsen, L. H., Martinussen, T. and Pedersen, H. D. (2003): Early echocardiographic predictors of myxomatous mitral valve disease in Dachshunds. Vet. Rec. 152, 293-297.

Patterson, D. F. (1968): Epidemiologic and genetic studies of congenital heart disease in the dog. Circ. Res. 23, 171-202.

Patterson, D. F., Haskins, M. E. and Schnarr, W. R. (1981): Hereditary dysplasia of the pulmonary valve in Beagle dogs. Pathologic and genetic studies. Am. J. Cardiol. 47, 631-641.

Pedersen, H. D., Häggström, J., Falk, T., Mow, T., Olsen, L. H., Iversen, L. and Jensen, A. L. (1999a): Auscultation in mild mitral regurgitation in dogs: Observer variation, effects of physical maneuvers, and agreement with color Doppler echocardiography and phonocardiography. J. Vet. Intern. Med. 13, 56-64.

Pedersen, H. D., Lorentzen, K. A. and Kristensen, B. Ø. (1999b): Echocardiographic mitral valve prolapse in Cavalier King Charles Spaniels: epidemiology and prognostic significance for regurgitation. Vet. Rec. 144, 315-320.

R Core Team (2013): R: A Language and Environment for Statistical Computing. R Foundation for Statistical Computing, Vienna, Austria. URL http://www.R-project.org/.

Sisson, D. and Ettinger, S. J. (1999): The physical examination. In: Fox, P. R., Moïse, N. S. and Sisson, D. (eds) Textbook of Canine and Feline Cardiology. Principles and Clinical Practice. 2nd edition. W. B. Saunders, Philadelphia. pp. 46-64.

Swenson, L., Häggström, J., Kvart, C. and Juneja, R. K. (1996): Relationship between parental cardiac status in Cavalier King Charles spaniels and prevalence and severity of chronic valvular disease in offspring. J. Am. Vet. Med. Assoc. 208, 2009-2012.

Szilvási, V., Vörös, K., Manczur, F., Reiczigel, J., Novák, I., Máthé, Á. and Fekete, D. (2013): Comparison of traditional and sensor-based electronic stethoscopes in Beagle dogs. Acta Vet. Hung. 61, 19-29.

Terzo, E., Di Marcello, M., McAllister, H., Glazier, B., Lo Coco, D., Locatelli, C., Palermo, V. and Brambilla, P. G. (2009): Echocardiographic assessment of 537 dogs with mitral valve prolapse and leaflet involvement. Vet. Radiol. Ultrasound 50, 416-422.

Thrusfield, M. V., Aitken, C. G. G. and Darke, P. G. G. (1985): Observation on breed and sex in relation to canine heart valve incompetence. J. Small Anim. Pract. 26, 709-717.

Tou, S. P., Adin, D. B. and Estrada, A. H. (2006): Echocardiographic estimation of systemic systolic blood pressure in dogs with mild mitral regurgitation. J. Vet. Intern. Med. 20, 1127-1137.

Trafny, D. J., Freeman, L. M., Bulmer, B. J., MacGregor, J. M., Rush, J. E., Meurs, K. M. and Oyama, M. A. (2012): Auscultatory, echocardiographic, biochemical, nutritional, and environmental characteristics of mitral valve disease in Norfolk terriers. J. Vet. Cardiol. 14, 261-267.

Vörös, K., Bonnevie, A. and Reiczigel, J. (2012): Comparison of conventional and sensor-based electronic stethoscopes in detecting cardiac murmurs of dogs. Tierärztl. Praxis 40, 103-111.

Vörös, K., Hetyey, Cs., Reiczigel, J. and Nagy Czirok, G. (2009): M-mode and two-dimensional echocardiographic reference values for three Hungarian dog breeds: Hungarian Vizsla, Mudi and Hungarian Greyhound. Acta Vet. Hung. 57, 217-227.

Vörös, K., Nolte, I., Hungerbühler, S., Reiczigel, J., Ehlers, J. P., Tater, G., Mischke, R., Zimmering, T. and Schneider, M. (2011): Sound recording and digital phonocardiography of cardiac murmurs in dogs by using a sensor-based electronic stethoscope. Acta Vet. Hung. 59, $23-35$.

Ware, W. A. (2007): Acquired valve diseases. In: Ware, W. A. (ed.) Cardiovascular Disease in Small Animal Medicine. Manson Publishing Ltd., London. pp. 263-279. 